\section{BNREL}

\section{$S D G E$}

A Sempra Energy utility

\section{Background}

Numerous distribution network analysis, monitoring, and control applications require accurate phase connectivity information in the system models. Existing phase identification techniques that estimate phase connectivity work well in distribution feeders that have low or no photovoltaic (PV) generation; however, they fail to identify photovoltaic (PV) generation; however, they fail to identify is present. This work addresses the phase identification is present. This work addresses the phase identification
problem in the presence of high PV generation using problem in the presence
statistical analysis methods.

\section{Feeder Characteristics}

- 12-kV feeder with a peak load of $10.3 \mathrm{MW}$ One substation load tap changer, three fixed capacitor banks for voltage regulation

- More than 4,200 single-phase nodes

- Distributed PV generation of approx. $30 \%$ relative to peak load.

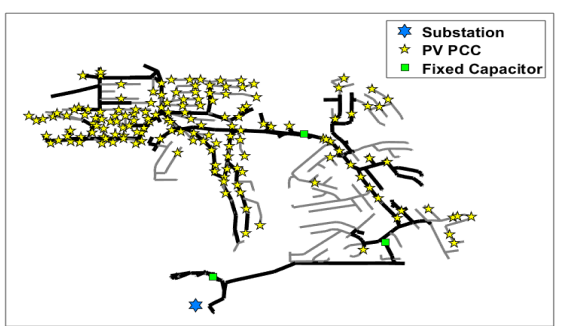

Figure 1. Topology of the distribution feeder

\section{Synthetic AMI Data Generation}

Synthetic AMI data are generated using a quasi-static time-series (QSTS) simulation of the feeder model in OpenDSS with a 1-min time step resolution.

The featured data from the QSTS are averaged over 5 -min intervals to mimic the data recordings of AM meters. The resulting data are used for data analysis.

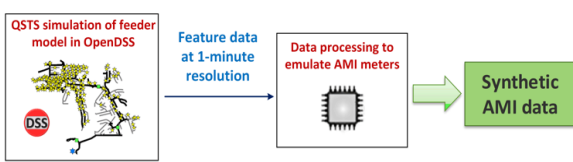

Figure 2. Synthetic AMI data generation process

\title{
Considerations for AMI-Based Operations for Distribution Feeders
}

Harsha Padullaparti ${ }^{1}$, Santosh Veda ${ }^{1}$, Surya Dhulipala ${ }^{3}$, Murali Baggu ${ }^{1}$, Tom Bialek², Martha Symko-Davies ${ }^{1}$

- Actual load and PV profile measurement data received from SDG\&E as shown Figure 3 are used for the QSTS simulation of the OpenDSS model.

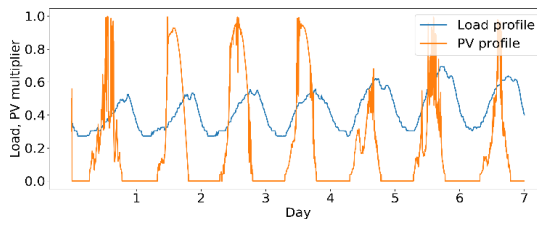

Figure 3. Load and PV profiles of 1-week period

\section{Scenario}

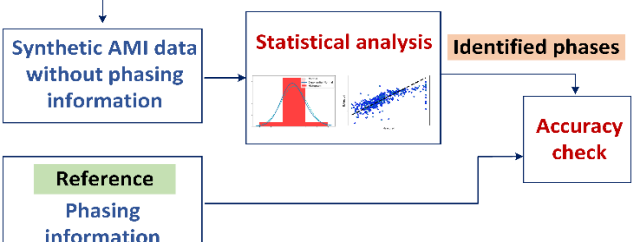

information

Figure 4. Phase identification through statistical analysis of AMI data

Several scenarios are created by varying PV penetration levels and AMI data resolutions, and the corresponding data are stored in different data sets.

The AMI data set of a given scenario is then passed to the statistical analysis module as input to apply multiple statistical methods.

The phase identification results from this module ar compared with the actual phasing information in the simulation model stored separately to check the accuracy of results.

\section{Phase Identification by Regression}

- Initially, multiple linear regression (MLR) analysis of average voltage and active power measurement dat of the nodes and substation with (1) is performed:

$$
V_{\text {sub }}=k_{0}+k_{1} V_{n}+k_{2} P_{n}+k_{3} P_{\text {sub }}
$$

Accurate phase identification results are accomplished with this approach only when the PV generation levels are low.

- Next, AMI data generated by including $30 \%$ existing PV generation are used for the MLR. The predicted substation voltages using the MLR model showed errors.

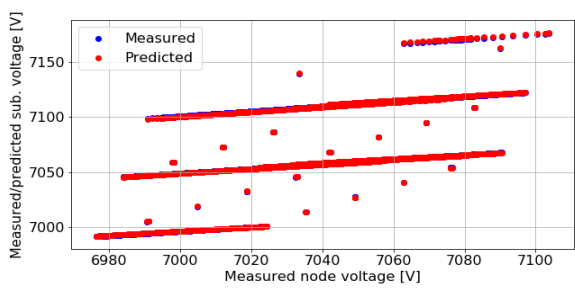

Figure 5. Predicted substation voltages with MLR accurately matching measured values without PV

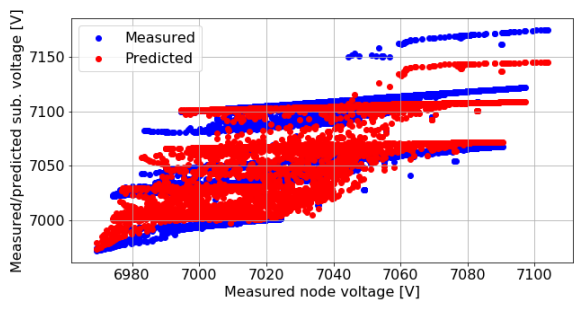

Figure 6. Errors in the predicted substation voltage using the MLR model in the presence of $30 \% \mathrm{PV}$

The prediction errors are primarily caused by not capturing the PV generation in terms of measurable features; thus, the regression models formed using (1) become inaccurate

\section{Sensitivity to Various Parameters}

(A) Data window length: Variation of data window engths used for analysis showed that all the methods except Method 4 could identify all the phases correctly with only a data window length of 3 hours.

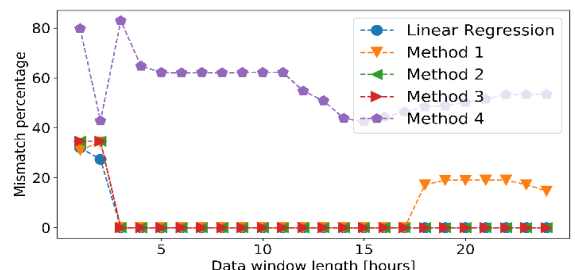

Data window length [hours]
Figure 7. Sensitivity of phase identification accuracy to data window length

Analysis was repeated with $\sim 30 \%$ existing PV generation in feeder. Only Method 4 provided accurate results for all data window lengths.

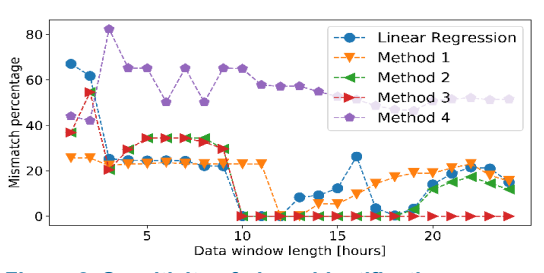

Figure 8. Sensitivity of phase identification accuracy to data window length

(B) PV penetration level: PV penetration levels are varied from $0 \%$ to $100 \%$ relative to peak load to create corresponding data sets. The analysis on these data sets showed that only Method 4 provided accurate results for all the PV penetration levels.

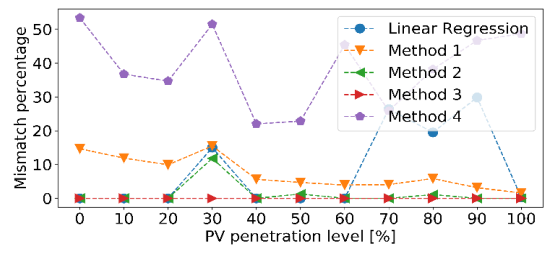
Figure 9. Sensitivity of phase identification accuracy to
PV penetration level

(C) Data resolution: Data analysis on synthetic AM (C) Data resolution: Data analysis on synthetic AMI
data at different resolutions showed that the accuracy did not vary much on the considered data sampling did not vary much on the considered data sampling
intervals. Multiple data resolutions were generated by averaging the 1 -min data at different intervals.

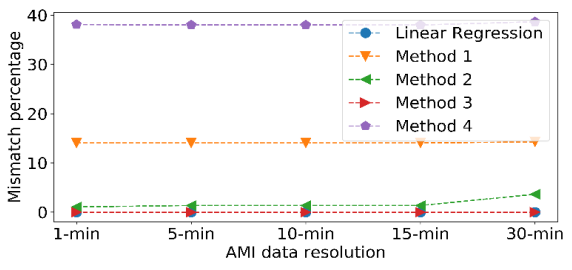

Figure 10. Sensitivity of phase identification accuracy to AMI data resolution

\section{Conclusion}

Existing phase identification techniques work well in the distribution feeders having low or no PV generation but fal when significant PV generation is present. In this work, the phase identification problem in the presence of high PV generation is addressed, and insights into AMI data requirements are provided. 